EPJ Web of Conferences 45, 01025 (2013)

DOI: $10.1051 /$ epjconf/20134501025

(C) Owned by the authors, published by EDP Sciences, 2013

\title{
Velocity profiles in idealized model of human respiratory tract
}

\author{
J. Elcner ${ }^{1, a}$, J. Jedelsky $^{1}$, F. Lizal $^{1}$, and M. Jicha ${ }^{1}$ \\ ${ }^{1}$ Brno University of Technology, Department of Thermodynamics and Environmental Engineering, Technicka 2896/2, \\ Brno, Czech Republic, yelcne00@stud.fme.vutbr.cz
}

\begin{abstract}
This article deals with numerical simulation focused on velocity profiles in idealized model of human upper airways during steady inspiration. Three regimes of breathing were investigated: Resting condition, Deep breathing and Light activity which correspond to most common regimes used for experiments and simulations. Calculation was validated with experimental data given by Phase Doppler Anemometry performed on the model with same geometry. This comparison was made in multiple points which form one cross-section in trachea near first bifurcation of bronchial tree. Development of velocity profile in trachea during steady inspiration was discussed with respect for common phenomenon formed in trachea and for future research of transport of aerosol particles in human respiratory tract.
\end{abstract}

\section{Introduction}

Breathing is phenomenon necessary for every mammal on planet Earth. During the breathing cycle there is a transfer of vital gases between environment and bloodstream. While the air during the inspiration carries the oxygen, which oxidizes the blood, during the expiration is carbon dioxide removed out of the bloodstream through respiratory system to the environment. This article deals with study of steady inspiration, where air flows from mouth nozzle through mouth cavity to larynx and then leads to trachea and bronchial tree and its aimed primary to area around trachea, where every inhaled air passes through. During the inspiration we can observe many phenomenons in tracheobronchial region, which plays significant role with assessing of transport and deposition of particles in human respiratory tract. Most of these phenomenons were previously studied and described. Research in this article is trying to establish to the formerly known studies which was focused to development of airflow in trachea and surrounding regions. Similar research on model of idealised geometry of oropharynx where geometry which ends in upper part of trachea was used was done by Heenan [1]. Unfortunately, this research cannot give us information about forming of velocity profiles in whole trachea. Investigation of respiratory system constriction influence to airflow in tracheobronchial region was made by Lin et al. [2]. In theirs study, comparison of two different cases of prescription of boundary condition was performed. First case contains model of human upper airways with mouth cavity, pharynx, larynx and tracheobronchial tree, second case contains tracheobronchial tree only. On the inlet to both cases, the velocity boundary condition was prescribed. Result of the comparison was necessity of inclusion of larynx into geometry model as necessary part which forms the air flow fields. Airflow in the trachea itself is further affected on its tail formed by bifurcation, where bronchial tree is divided to left and right part of lungs. Influence of this bifurcation on the shape of velocity profile was studied by Freitas and Schröder [3] and also influence of shape of the bifurcation was investigated by Martonen [4], who made an analysis on four kinds of bifurcation and prove the affect of carinal ridge on airflow fields in following generation of branching of bronchial tree. For the purposes of this study a model [5] which enables achieve more accurate measurements by Phase-Doppler Anemometry was used. This model also fulfil all prerequisites above, because it contains larynx and its bifurcations are made with respect to realistic model of bronchial tree. Comparison of calculations and experimental data was made for most commonly used breathing cycles [6].

\section{Methods}

\subsection{Model}

The model (see figure 1), used during experiments and numerical simulations is based on realistic geometry model of human upper respiratory tract [5]. Geometry of the model was simplified with the purpose for reach more accurate results of experiments. This was achieved by replacing realistic geometry of tracheobronchial area of 


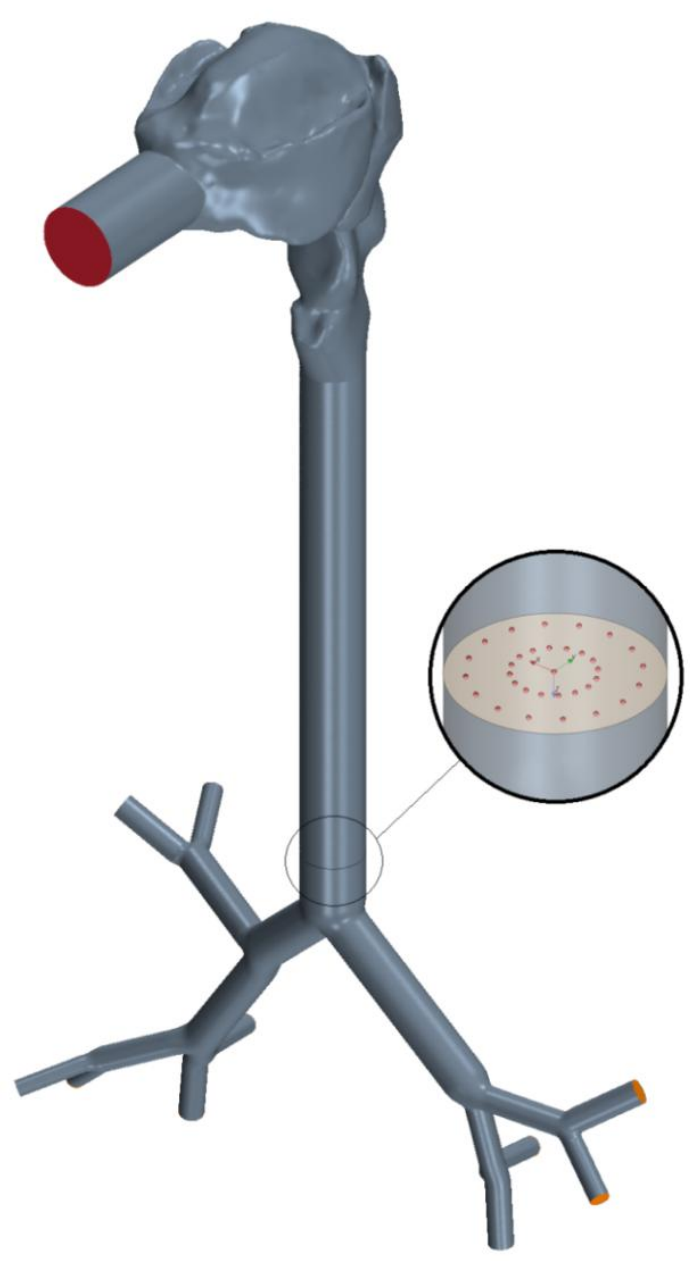

Fig. 1. Idealized model of human respiratory tract

model by simplified geometry based on tubes of circular cross-section, which can be formed by thin walled glass tube on physical model used for experiments. Thanks to these assumptions the optically transparent model, enabling to perform measurements using P/DPA in arbitrary part of trachea and branches of bronchial tree, was developed. The model contains realistic geometry of mouth nozzle, mouth cavity and larynx, which was made by rapid prototyping. This part was mounted with thin walled glass tube of $15.2 \mathrm{~mm}$ in diameter which represents trachea. Diameter of idealized trachea was derived from realistic geometry, based on similarity of volumes and lengths of realistic and idealized trachea. Bottom of trachea is equipped idealized model of bronchial tree where bifurcations respect the branching angles on realistic geometry and they were made by rapid prototyping, and branches were made by thin walled glass tubes as trachea.

\subsection{Experimental setup}

The experiment was performed using P/DPA technique. 1D PDA (Dantec Dynamics) with Ar-Ion+ Laser ILT 5500A-00 (max. power $300 \mathrm{~mW}$ ) was used for measurement of time-resolved flow velocity at multiple points of the model for three different flow rates which corresponds to frequently used breathing regimes. The PDA focal length of both the transmitter and receiver was $310 \mathrm{~mm}$; 1st order light refraction and scattering angle $45^{\circ}$ was used. Tracer particles of di-2-ethylhexyl Sebacate (DEHS) with $3 \mu \mathrm{m}$ in diameter were produced by a condensation monodisperse aerosol generator (CMAG TSI 3475); they were mixed with air in a static mixer and led to the model. The flow rates for used breathing regimes are defined in Table 1 . The measurement was done in 33 points located in one crosssection in trachea, $23 \mathrm{~mm}$ above carinal ridge of first bifurcation. A velocity component in direction perpendicular to the cross-section of the trachea (expected direction of air flow in the model) was measured. The particle velocity is considered to match with air flow velocity due to low Stokes number (less than 0.02). More information about experimental setting can be found in [7].

Table 2. Breathing regimes used for comparison.

\begin{tabular}{|l|l|l|l|}
\hline \multicolumn{1}{|c|}{ Activity } & $\begin{array}{c}\text { Flow } \\
\text { rate } \\
\mathrm{Q}[1 / \mathrm{min}]\end{array}$ & $\begin{array}{c}\text { Inlet } \\
\text { velocity } \\
\mathrm{u}_{\mathrm{i}}[\mathrm{m} / \mathrm{s}]\end{array}$ & $\begin{array}{c}\text { Reynolds } \\
\text { number } \\
\mathrm{Re}\end{array}$ \\
\hline Resting cond. & 15 & 0.79 & 1404 \\
\hline Deep breathing & 30 & 1.58 & 2809 \\
\hline Light activity & 60 & 3.16 & 5618 \\
\hline
\end{tabular}

\subsection{Numerical setup}

Calculation was performed using StarCCM+ solver. Polyhedral volume mesh with prismatic layer containing approximately 1500000 cells was generated on the model geometry. The model was situated regarding the position during the experiment which corresponds to position of the upper airways in human body. Axis of trachea was parallel to $\mathrm{Z}$ axis of coordinate system and $\mathrm{X}$ axis head towards right branch of bronchial tree. Velocity boundary condition, corresponding to the investigated breathing regime (see Table 1) was set on the inlet to the model (mouth nozzle) and pressure outlet boundary condition was prescribed on the terminal section of branches in last generation of models bronchial tree. The problem was calculated using unsteady RANS with komega turbulence model with low Reynolds conditions treatment. All points measured in experiment were monitored during calculation. Position of the points in the model is visible at figure 1.

\section{Results}

Results confirmed the expecting phenomenons in trachea region. Air entering through the nozzle at mouth cavity adheres to the tongue which leads to a change in pressure in mouth cavity and increases the value of velocity of airflow. Air then follows with copying the shape of tongue and entering to the larynx where airflow velocity increasing as a result of constriction of airway geometry. Thanks to the specific shape of larynx, the air enters to the trachea in direction which is not parallel to the axis of trachea. This direction, as written in introduction, is 
characteristic for laryngeal jet and head to anterior region of trachea (axis -Y). Since, the trachea on our model is idealized and their shape is simplified to cylinder, we expect the behaviour of air flow as can be seen during airflow in a pipe and standard dependence on Reynolds number can be expected.

\subsection{Airflow profiles}

In the case of resting condition, the value of Reynolds number is $\operatorname{Re}=2400$, which corresponds to transitional flow and expected shape of velocity profile could be parabolic. Contours on figure 2, which contains comparison of velocity field during resting conditions indicate us the shape of the profile which is more similar to turbulent profile than laminar. Contours of constant velocities are close together near the wall of the model and then in the middle of the cross-section, there is a large area of constant velocity. There is also visible on the picture, that the flow is not symmetrical by any of axes of coordinate system. Because of the peak of velocity profile is situated in anterior region of the model and deflects from the axis of trachea to the right side of the bronchial tree, it is visible that the flow is not completely developed. Experiments and calculations were performed in bottom part of trachea (9/10 of its length) which means that the laryngeal jet affects airflow in whole trachea and following bronchial tree. There are similar results during higher breathing regimes, which is apparent on figures 3 and 4 .

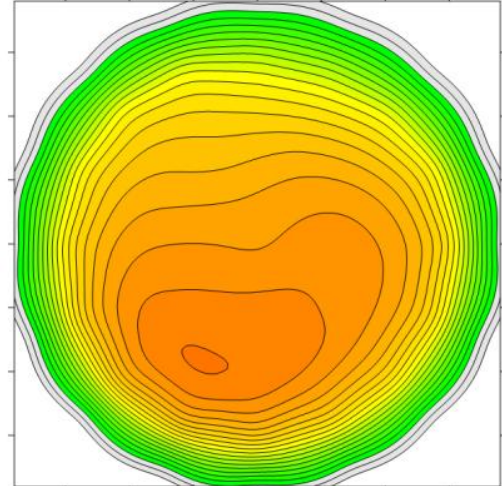

(a) Experiment

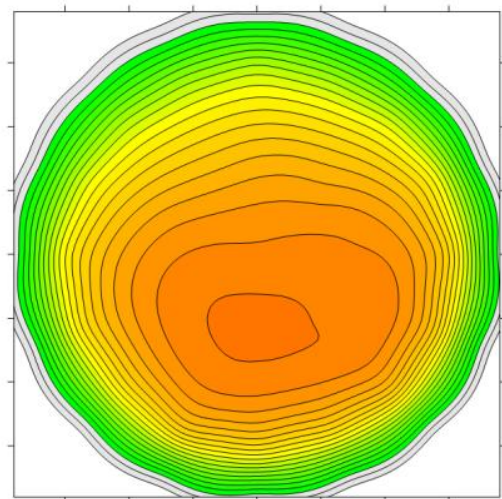

(b) Calculation

Fig. 2. Flow field in trachea, 151/min

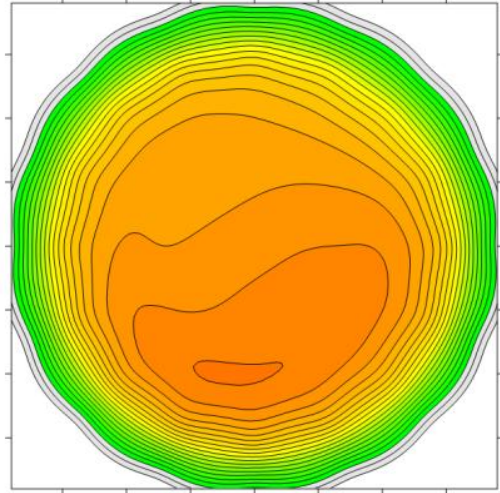

(a) Experiment

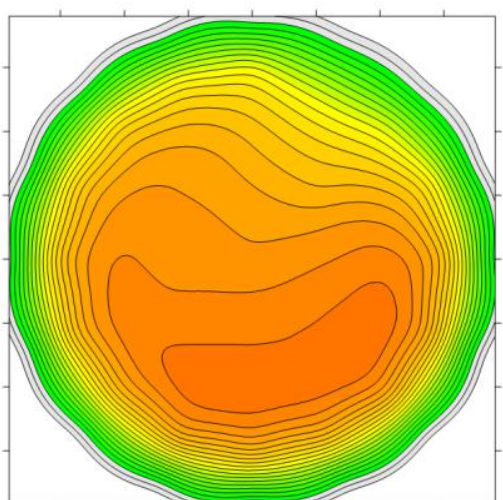

(b) Calculation

Fig. 3. Flow field in trachea, $301 / \mathrm{min}$

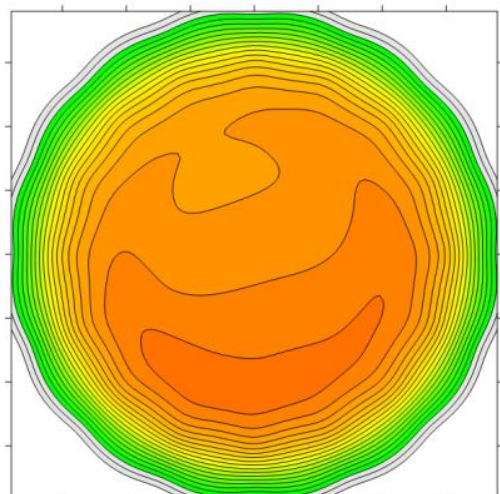

(a) Experiment

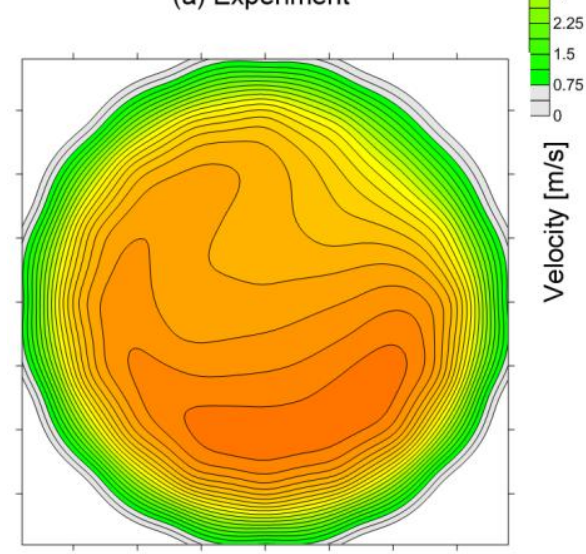

(b) Calculation 


\subsection{Numerical - Experimental comparison}

Comparison between experiment and calculation was done by program Surfer in all 33 measured points, by using kriging method, which can be applied for large amount of situations and it is usable wherever it can be found a space-oriented variability of variable. For higher accuracy of comparison, the method was applied not only to experimental data, but also on calculated values. Experimental data had to be correlated for reasons of low accuracy of setting of flowmeter before the inlet to the model. Comparison on figures 2-4 shows the qualitative agreement where similar direction of axis of flow is visible.

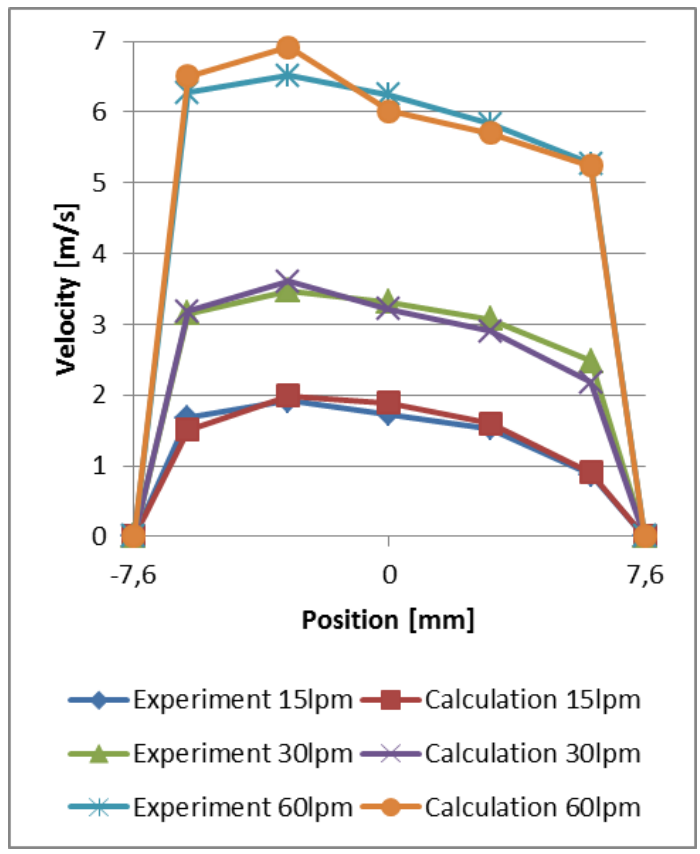

Fig. 5. Velocity profiles in trachea, plane $\mathrm{XZ}$

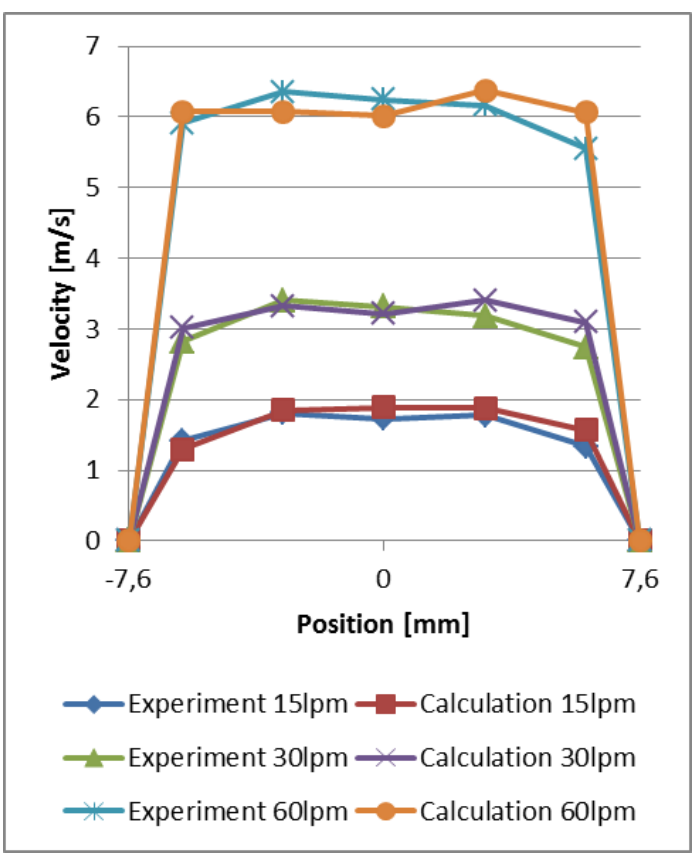

Fig. 6. Velocity profiles in trachea, plane YZ
For better comparison, charts showing velocity profile in planes XZ and YZ (see figure 5 and 6) were made. These charts show us good agreement in axis of trachea for all three breathing regimes but increasing level of uncertainty in near-wall area during higher values of airflow. This can be caused by selected numerical method and especially model of turbulence.

\section{Conclusions}

This work shows good agreement between experimental and calculated data from idealized model of human trachea. Differences in values should be corrected by selection of suitable numerical model. Analysed phenomenas in this area will help us with future numerical research of transport and deposition of aerosols in human upper airways.

\section{Acknowledgment}

This work was supported by project FSI-S-11-6.

\section{References}

1. A. F. Heenan, E. Matida, A. Pollard, W.H. Finlay, Experiments in fluids 35, 70-84 (2003)

2. Ch. - L. Lin, M. H. Tawhai, G. McLennan, E.A. Hoffman, Respiratory Physiology \& Neurobiology 157, 295-309 (2007)

3. R. - K. Freitas, W. Schröder, Journal of Biomechanics 41, 2446-2457 (2008)

4. T. B. Martonen, Y. Yang, Z. Q. Xue, Aerosol science and technology 21, 119 - 136 (1994)

5. F. Lizal, J. Elcner, P. Hopke, J. Jedelsky, M. Jicha, Proceedings of the institution of mechanical engineers Part $H$ - Journal of engineering in medicine 226, 197-207 (2011)

6. C. Kleinstreuer, Z. Zhang, International Journal of Multiphase Flow 29, 271-289 (2002)

7. J. Jedelsky, M. Jicha, F. Lizal, J. Elcner, 9th Intl. ERCOFTAC Symposium on Engineering Turbulence Modeling and Measurements , (2012) 